\title{
Positive Feedback Loop of Autocrine BDNF from Microglia Causes Prolonged Microglia Activation
}

\author{
Xin Zhang Lulu Zeng Tingting $\mathrm{Yu}$ Yongming $\mathrm{Xu}$ Shaofeng $\mathrm{Pu}$ \\ Dongping Du Wei Jiang \\ Pain Management Center, Shanghai Jiao Tong University Affiliated Sixth People's Hospital, Shanghai, \\ China
}

\author{
Key Words \\ BDNF • ATP • Microglia • Pain
}

\begin{abstract}
Background/Aims: Microglia, which represent the immune cells of the central nervous system (CNS), have long been a subject of study in CNS disease research. Substantial evidence indicates that microglial activation functions as a strong neuro-inflammatory response in neuropathic pain, promoting the release of proinflammatory cytokines, such as tumor necrosis factor (TNF)- $\alpha$. In addition, activated microglia release brain-derived neurotrophic factor (BDNF), which acts as a powerful cytokine. In this study, we performed a series of in vitro experiments to examine whether a positive autocrine feedback loop existed between microglia-derived BDNF and subsequent microglial activation as well as the mechanisms underlying this positive feedback loop. Methods: Because ATP is a classic inducer of microglial activation, firstly, we examined ATP-activated microglia in the present study. Secondly, we used TrkB/Fc, the BDNF sequester, to eliminate the effects of endogenous BDNF. ATP-stimulated microglia without BDNF was examined. Finally, we used exogenous BDNF to further determine whether BDNF could directly activate BV2 microglia. In all experiments, to quantify BV2 microglia activation, the protein levels of $C D 11 b$, a microglial activation marker, were measured by western blot. A Transwell migration assay was used to examine microglial migration. To assess the synthesis and release of proinflammatory cytokines, western blot was used to measure BDNF synthesis, and ELISA was used to quantify TNF- $\alpha$ release. Results: In our present research, we have observed that ATP dramatically activates microglia, enhancing microglial migration, increasing the synthesis of BDNF and up-regulating the release of TNF- $\alpha$. Microglial activation is inhibited
\end{abstract}

X. Zhang, L. Zeng, D. Du and W. Jiang contributed equally to this work. 


\section{Cellular Physiology and Biochemistry}

Cell Physiol Biochem 2014;34:715-723

DOI: $10.1159 / 000363036$

Publisned online: August 18, 2014

(c) 2014 S. Karger AG, Basel

www.karger.com/cpb

Zhang et al.: Autocrine Activation of Microglia by BDNF

following the sequestration of endogenous BDNF, resulting in impaired microglial migration and decreased TNF- $\alpha$ release. Furthermore, exogenous BDNF can also activate microglia to subsequently enhance migration and increase TNF- $\alpha$ release. Conclusion: Therefore, we suggest that microglial activation increases the synthesis of BDNF and that the release of BDNF can, in turn, activate microglia. A positive autocrine BDNF feedback loop from microglia may contribute to prolonged microglial activation.

Copyright (C) 2014 S. Karger AG, Basel

\section{Introduction}

Neuropathic pain represents a particularly frustrating problem for researchers due to its unclear underlying mechanisms and its resistance to routine treatment [1]. In previous decades, our understanding of pain has focused solely on the role of neurons, whereas and the neighboring microglia have been considered simply resident macrophages of the central nervous system (CNS). However, recent studies indicate that microglia can function as key players in the modulation of neuronal network excitability [2]. Furthermore, neuropathic pain pathogenesis depends on both changes in the activity of neuronal systems as well as microglia activation [3]. During the pathogenesis of neuropathic pain, resident microglia become activated [4], exhibiting up-regulation of the expression of cell surface receptors (including purinergic receptors and CD11b) [5, 6], enhanced migratory ability [7] and proinflammatory cytokine release (including tumor necrosis factor (TNF)- $\alpha$ and brain-derived neurotropic factor (BDNF)] [8, 9], which can enhance synaptic transmission. Together, these changes can accelerate the development of neuropathic pain.

BDNF, one of the most important neurotrophins, contributes to many aspects of CNS function, including cell differentiation, neuronal survival and migration, dendritic arborization, synaptogenesis and activity-dependent forms of synaptic plasticity [10]. Recently, a growing body of evidence has indicated that BDNF may also represent a key molecule mediating microglia-neuron signaling via its high-affinity receptor, tropomyosin-related kinase $B$ (TrkB) [11]. In neuropathic pain conditions, adenosine triphosphate (ATP) can activate microglia via P2X4 purinoceptors (P2X4Rs), which then up-regulate the synthesis and release of BDNF [12]. Both the release and expression of BDNF are $\mathrm{Ca}^{2+}$-dependent and mediated by the activation of p38 mitogen-activated protein kinase (MAPK), a classical kinase that has been implicated in neuropathic pain [12]. Because TrkB is widely expressed in many cell types, BDNF exhibits multiple biophysical effects in the CNS. BDNF mediates central sensitization in lamina II by altering inhibitory neurotransmission [13]. Our previous study demonstrated that BDNF can induce astrocyte activation, which can lead to the development of neuropathic pain [14]. Notably, BDNF may also act on microglia; the study by Zhou et al. [15] suggests that increased BDNF/TrkB signaling in the dorsal horn of the spinal cord may activate microglia, thereby contributing to peripheral nerve injury-induced neuropathic pain. These skillful experiments were performed in a rat model, but direct evidence of BDNF stimulation on microglia remains lacking. In this study, we performed a series of in vitro experiments to examine whether a positive autocrine feedback loop existed between microglia-derived BDNF and subsequent microglial activation as well as the mechanisms underlying this positive feedback loop.

\section{Materials and Methods}

Reagents

Phosphate-buffered saline (PBS) and ATP were purchased from Sigma-Aldrich Chemical (St. Louis, MO, USA). TrkB/Fc chimeric protein and recombinant human BDNF were purchased from R\&D (Minneapolis, MN, USA) and Prospec (Ness-Ziona, Israel), respectively, and dissolved in PBS. Fetal bovine serum (FBS) 


\section{Cellular Physiology and Biochemistry}

Cell Physiol Biochem 2014;34:715-723

DOI: $10.1159 / 000363036$

Publisned online: August 18, 2014

(C) 2014 S. Karger AG, Basel

www.karger.com/cpb

and Dulbecco's Modified Eagle's medium (DMEM) were obtained from Gibco (Grand Island, NY, USA). AntiCD11b, anti-BDNF, anti- $\alpha$-Tubulin and anti-GAPDH antibodies were purchased from Santa Cruz Corp (Santa Cruz, CA, USA).

\section{Cell culture}

The BV2 murine microglial cell line was purchased from the Chinese Cell Resource Center (Institute of Basic Medical Science, Chinese Academy of Medical Sciences, School of Basic Medicine Peking Union Medical College, Peking, China). The cells were maintained in DMEM supplemented with 10\% FBS, 100 U/ $\mathrm{ml}$ penicillin and $100 \mu \mathrm{g} / \mathrm{ml}$ streptomycin (P/S) in a humidified incubator with $5 \% \mathrm{CO}_{2}$ at $37^{\circ} \mathrm{C}$.

\section{Western immunoblots}

Western blot analysis was performed to quantify the protein expression of BDNF and CD11b in BV2 cell culture using a standard protocol. Briefly, fresh cell samples were collected and homogenized at $4^{\circ} \mathrm{C}$ in 5 volumes of extraction buffer [50 mM Tris ( $\mathrm{pH} 7.4$ ), $150 \mathrm{mM} \mathrm{NaCl}, 1 \% \mathrm{NP}-40,0.5 \%$ sodium deoxycholate, $0.1 \%$ SDS, and protease inhibitor cocktail (Complete, Roche Diagnostics)] using a hand-held homogenizer. We measured the protein concentration using a BCA protein assay kit (Beyotime Corporation, Nanjing, China). After aliquots of protein $(30 \mu \mathrm{g})$ in SDS-PAGE protein loading buffer (Beyotime Corporation, Nanjing, China) were heated at $95^{\circ} \mathrm{C}$ for $10 \mathrm{~min}$, the proteins were separated using SDS-PAGE gels and transferred onto PVDF membranes (Millipore Corporation, Billerica, MA, USA). the membranes were incubated in blocking buffer (5\% milk in Tris-buffered saline [TBS] with $0.1 \%$ Tween 20 ) for $1 \mathrm{~h}$ at room temperature, followed by overnight incubation at $4^{\circ} \mathrm{C}$ with primary anti-BDNF (1:200; Santa Cruz Corporation, Santa Cruz, CA, USA), anti-CD11b (1:200; Santa Cruz Corporation, Santa Cruz, CA, USA) and anti- $\alpha$-Tubulin antibodies (1:1000; Santa Cruz Corporation, Santa Cruz, CA, USA). Subsequently, the membranes were washed three times in TBS and incubated with horseradish peroxidase-conjugated anti-rabbit IgG antibody (1:5000; Santa Cruz Corporation,) for $2 \mathrm{~h}$ at room temperature. After washing the membranes three times in TBS, immunoreactive blots were detected with enhanced chemoluminescence (Amersham Biosciences, Piscataway, NJ, USA) and visualized through X-ray film exposure (Kodak, Shanghai, China). We subsequently incubated the blots in stripping buffer (Beyotime Corporation, Nangjing, China) for $10 \mathrm{~min}$ at $4^{\circ} \mathrm{C}$ and reprobed the blots with an anti-GAPDH antibody (1:1000; Santa Cruz Corporation, Santa Cruz, CA, USA), which was used as the loading control. We determined the immunoreactive band intensity using Quantity One software (Bio-Rad, Hercules, CA, USA) and normalized the relative levels of BDNF against GAPDH and the relative levels of CD11b against $\alpha$-Tubulin; the data are presented as the BDNF/GAPDH and CD11b/ $\alpha$ Tubulin ratios.

\section{Migration assay}

Migration assays were performed using 24-well format, 8.0- $\mu$ m pore size Transwell inserts (Costar, NY, USA). BV2 cells with or without ATP were plated in the 24 -well plates $\left(4 \times 10^{5}\right.$ cells per well) for 24 $\mathrm{h}$. Prior to performing the migration assay, the cells were cultured with serum-free DMEM for $24 \mathrm{~h}$, and Basement Membrane Extract (BME) was coated with Lysine (Sigma-Aldrich Chemical, St. Louis, MO, USA). Approximately $4 \times 10^{5}$ cells in $200 \mu \mathrm{l}$ of serum-free medium were placed in the upper chamber, and $600 \mu \mathrm{l}$ of the same medium containing 10\% FBS was placed in the lower chamber. The plates were incubated for 24 $\mathrm{h}$ at $37^{\circ} \mathrm{C}$ in $5 \% \mathrm{CO}_{2}$. The inserts were subsequently removed and fixed in $4 \%$ paraformaldehyde for $10 \mathrm{~min}$, followed by staining with hematine for $10 \mathrm{~min}$. The cells on the upper sides of the filters were removed with cotton-tipped swabs. The migration of the BV2 microglia was quantified by counting the number of cells that migrated through the membrane to the other side. The images were selected from eight random fields for each treatment with a microscope at $50 \times$ magnification, and the number of migrating cell was quantified. Each experiment was repeated a minimum of three times.

Enzyme-Linked Immunosorbent Assay

BV2 cells were cultured in triplicate in a 48-well plate. The supernatants of each treatment were collected to quantify the levels of TNF- $\alpha$ using a standard sandwich ELISA (R\&D Systems, Minneapolis, MN, USA). 


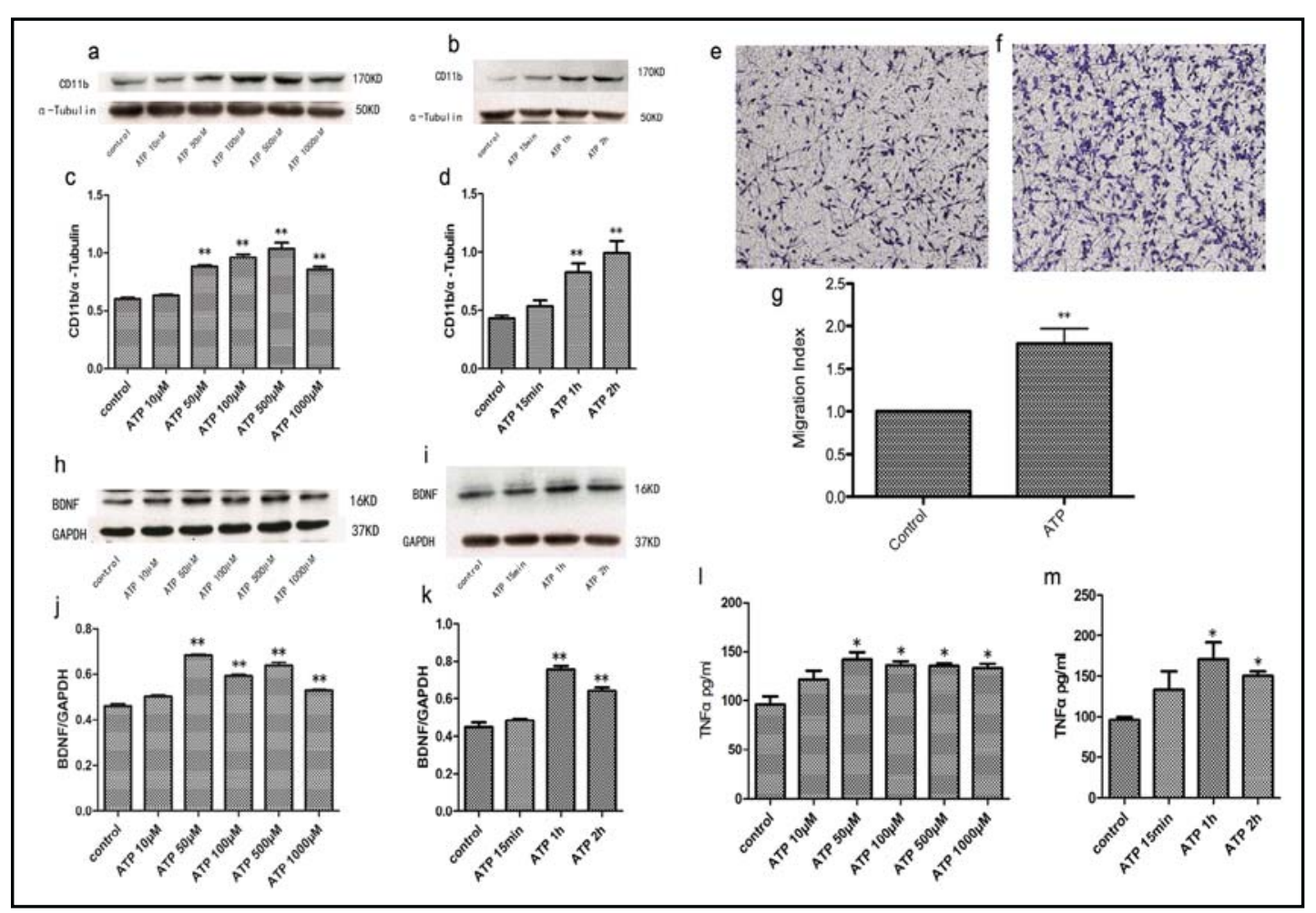

Fig. 1. ATP activates microglia, enhancing microglial migration, BDNF synthesis and the release of TNF- $\alpha$. a Immunoblots of CD11b and $\alpha$-Tubulin in BV2 microglia incubated with varying concentrations of ATP. b Immunoblots of CD11b and $\alpha$-Tubulin in BV2 microglia incubated with $500 \mu \mathrm{mol} / \mathrm{l}$ of ATP for varying lengths of time. c, d ATP increased the protein levels of CD11b on BV2 microglia in a dose- and time-dependent manner $\left({ }^{* *} p<0.01\right.$, vs. control). e, f Image of migrating BV2 microglia incubated with (right) or without (left) ATP for $24 \mathrm{~h}$. g The migration index was used to quantify the number of migrating cells relative to the control $\left({ }^{* *} p<0.01\right.$, vs. control). h Immunoblots of BDNF and GAPDH in BV2 microglia incubated with ATP at varying concentrations. i Immunoblots of BDNF and GAPDH in BV2 microglia incubated with 500 $\mu \mathrm{mol} / \mathrm{l}$ of ATP for varying lengths of time. $\mathrm{j}$, $\mathrm{k}$ ATP increased the protein levels of BDNF in BV2 microglia in a dose- and time-dependent manner ( ${ }^{* *} p<0.01$, vs. control). l Concentrations of TNF- $\alpha$ in the supernatants of BV2 microglia incubated with ATP at varying concentrations as measured by ELISA. $m$ Concentrations of TNF- $\alpha$ in the supernatants of BV2 microglia incubated with $500 \mu \mathrm{mol} / \mathrm{l}$ of ATP for varying lengths of time as measured by ELISA. ATP enhanced the release of TNF- $\alpha$ in BV2 microglia in a dose- and time-dependent manner $\left({ }^{*} p<0.05\right.$, vs. control).

\section{Statistical analysis}

We analyzed the data with Prism 5 (Version 5.0c, GraphPad Software Inc., San Diego, CA, USA), and the results are presented as the mean \pm SEM. All experiments were repeated a minimum of 3 times. For statistical analyses, western blot, migration assays and cytokine data were analyzed using one-way ANOVA followed by multiple comparisons using the Student-Newman-Keuls post hoc test. In all comparisons, differences were considered significant at $p<0.05$.

\section{Results}

ATP activates microglia, enhancing microglial migrating, BDNF synthesis and TNF- $\alpha$ release

Because ATP is a classic inducer of microglial activation, we examined ATP-activation of microglia in the present study. To quantify BV2 microglia activation, the protein levels of CD11b, a microglial activation marker, were measured by western blot. First, the cells 


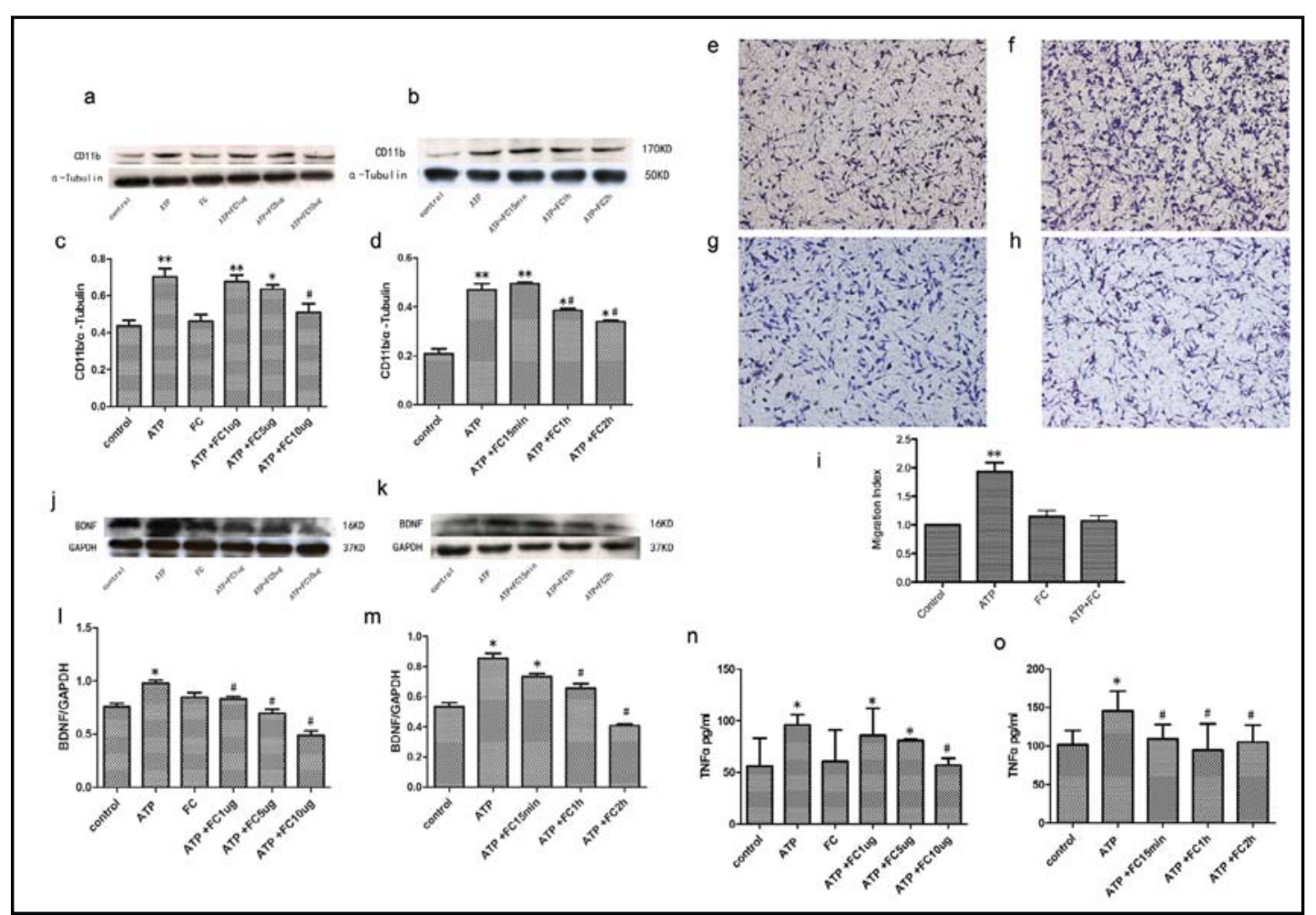

Fig. 2. TrkB/Fc, the BDNF sequester, inhibits ATP-induced microglial activation, inhibits migration and attenuates the release of TNF- $\alpha$. a, b Immunoblots of CD11b and $\alpha$-Tubulin in BV2 microglia. Following pre-incubation with TrkB/Fc, BV2 microglia were incubated with ATP. c, d When BV2 microglia were preincubated with TrkB/Fc, ATP-induces increases in CD11b protein levels were inhibited $\left({ }^{* *} p<0.01,{ }^{*} p<0.05\right.$, vs. control; ${ }^{*} p<0.05$, vs. ATP). e Image of migrating BV2 microglia following incubation with vehicle for $24 \mathrm{~h}$. f Image of migrating BV2 microglia following incubation with ATP for $24 \mathrm{~h}$. g Image of migrating BV2 microglia following incubation with TrkB/Fc for $24 \mathrm{~h}$. h Image of migrating BV2 microglia following incubation with ATP plus TrkB/Fc for $24 \mathrm{~h}$. i The migration index was used to quantify the number of migrating cells relative to the control $\left({ }^{* *} p<0.01\right.$, vs. control). j, $\mathrm{k}$ Immunoblots of BDNF and GAPDH in BV2 microglia. Following pre-incubation with TrkB/Fc, BV2 microglia were incubated with ATP. l, m Pre-incubation with TrkB/Fc inhibited the ATP-induced increase in BDNF protein levels in BV2 microglia ${ }^{*} p<0.05$, vs. control; ${ }^{\#} p<0.05$, vs. ATP). $n$, o Concentration of TNF- $\alpha$ in the supernatants of BV2 microglia as measured by ELISA. Preincubation with TrkB $/ \mathrm{Fc}$ inhibited the ATP-dependent TNF- $\alpha$ release $\left({ }^{*} p<0.05\right.$, vs. control; ${ }^{\#} p<0.05$, vs. ATP).

were treated with varying concentrations of ATP $(50,100,500$, or $1000 \mu \mathrm{mol} / \mathrm{l})$ for $1 \mathrm{~h}$. ATP activated microglia in a dose-dependent manner ( $p<0.01$, Fig. $1 \mathrm{a}$ and $c)$. Next, the cells were incubated with $500 \mu \mathrm{mol} / \mathrm{l}$ of ATP for varying lengths of time $(15 \mathrm{~min}, 1 \mathrm{~h}$, or $2 \mathrm{~h}$ ). The microglia were activated by $500 \mu \mathrm{mol} / \mathrm{l}$ of ATP in a time-dependent manner $(p<0.01$, Fig. $1 \mathrm{~b}$ and d). Subsequently, a Transwell migration assay was used to examine microglial migration. BV2 cells with or without ATP $(500 \mu \mathrm{mol} / \mathrm{l})$ were plated in 24 -well plates for $24 \mathrm{~h}$. The number of cells that successfully migrated through the membrane in the ATP group was significantly higher than the migrating cells in the control group ( $p<0.01$, Fig. 1 e, f, and g). Finally, to assess the synthesis and release of proinflammatory cytokines, western blot was used to measure BDNF synthesis, and ELISA was used to quantify TNF- $\alpha$ release. As shown in Fig. 1, following activation of the BV2 microglia, both the protein levels of BDNF in cell cultures and the concentration of TNF- $\alpha$ in the supernatant were up-regulated in a dose- and time-dependent manner ( $p<0.01$, Fig. $1 \mathrm{~h}, \mathrm{l}, \mathrm{j}, \mathrm{k}, \mathrm{l}$, and $\mathrm{m}$ ). This series of experiments suggests that ATP activation of microglia results in increased BDNF production, enhanced microglial migration and up-regulation of TNF- $\alpha$ release. 


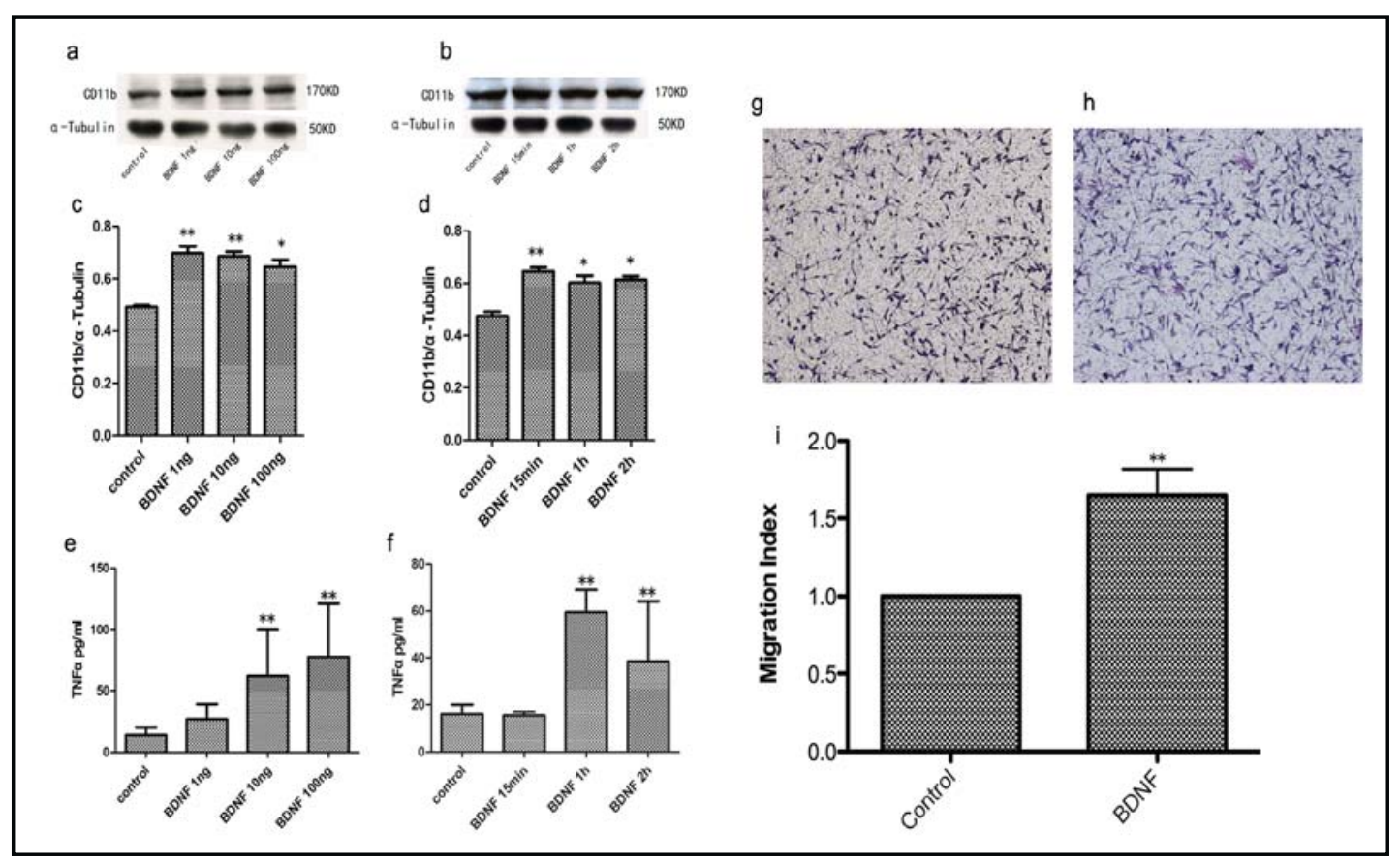

Fig. 3. Exogenous BDNF activates microglia, enhancing migration and increasing TNF- $\alpha$ release. a Immunoblots of $\mathrm{CD} 11 \mathrm{~b}$ and $\alpha$-Tubulin in BV2 microglia incubated with exogenous BDNF at varying concentrations. b Immunoblots of CD11b and $\alpha$-Tubulin in BV2 microglia incubated with $100 \mathrm{ng} / \mathrm{ml}$ of BDNF for varying lengths of time. c, d BDNF increased the protein levels of CD11b in BV2 microglia in a dose- and time-dependent manner ( ${ }^{* *} p<0.01$, vs. control). e, f Image of migrating BV2 microglia following incubation with (right) or without (left) BDNF for $24 \mathrm{~h}$. g The migration index was used to quantify the number of cells relative to the control ( ${ }^{* *} p<0.01$, vs. control). h Concentration of TNF- $\alpha$ in the supernatants of BV2 microglia incubated with BDNF at varying concentrations as measured by ELISA. m Concentrations of TNF- $\alpha$ in the supernatants of BV2 microglia incubated with $100 \mathrm{ng} / \mathrm{l}$ of BDNF for varying lengths of time as measured by ELISA. BDNF enhanced the release of TNF- $\alpha$ in BV2 microglia in a dose- and time-dependent manner $\left({ }^{*} p<0.05\right.$, vs. control).

$T r k B / F c$, the BDNF sequester, inhibits ATP-induced microglial activation, reduces migration and attenuates the release of TNF- $\alpha$

In this series of experiments, we used TrkB/Fc, the BDNF sequester, to eliminate the effects of endogenous BDNF. Prior to activation by ATP, BV2 microglia were pre-incubated with $\operatorname{TrkB} / \mathrm{Fc}(1,5$, or $10 \mu \mathrm{g} / \mathrm{l})$ for $1 \mathrm{~h}$. Microglial activation was quantified by measuring CD11b protein levels. As shown in Fig. 2, treatment with $10 \mu \mathrm{g} / \mathrm{l} \mathrm{TrkB} / \mathrm{Fc}$ prevented the ATP-dependent increase in CD11b protein levels in BV2 microglia. The effect of TrkB/Fc on ATP-dependent microglial activation was both dose- and time-dependent $(p<0.01$, Fig. 2a, $b$, c, and d). The number of migrating cells in the ATP plus TrkB/Fc group was significantly lower than the number of migrating cells in the ATP group. Pre-incubation with TrkB/Fc inhibited ATP-dependent increases in microglial migration ( $p<0.01$, Fig. 2e, f, g, h, and i). As demonstrated by the western blot and ELISA data, pre-incubation with TrkB/Fc attenuated the ATP-dependent release of TNF- $\alpha$ and synthesis of BDNF ( $p<0.01$, Fig. $2 \mathrm{j}, \mathrm{k}, \mathrm{l}, \mathrm{m}, \mathrm{n}$, and o). These experiments indicate that activated microglia can synthesize increased levels of BDNF; however, when the effects of endogenous BDNF are blocked, microglia activation is down-regulated, leading to the subsequent inhibition of microglial migration and proinflammatory factor release. 


\section{Cellular Physiology and Biochemistry}

Cell Physiol Biochem 2014;34:715-723

\begin{tabular}{l|l}
\hline DOI: $10.1159 / 000363036$ & (C) 2014 S. Karger AG, Basel
\end{tabular}

www.karger.com/cpb

Zhang et al.: Autocrine Activation of Microglia by BDNF

\section{Exogenous BDNF activates microglia, enhancing microglial migration and increasing} TNF- $\alpha$ release

In this series of experiments, we used exogenous BDNF to further determine whether BDNF can directly activate BV2 microglia. Exogenous BDNF increased the protein levels of CD11b on BV2 microglia in a dose- and time-dependent manner ( $p<0.01$, Fig. $3 a, b$, c, and d). The number of migrating cells in the BDNF group was greater than the number of migrating control cells, indicating that exogenous BDNF can enhance microglial migration $(p<0.01$, Fig. $3 \mathrm{~g}, \mathrm{~h}$, and i). Furthermore, the ELISA results revealed that exogenous BDNF can also upregulate the release of TNF- $\alpha(p<0.01$, Fig. $3 \mathrm{e}$ and f). Based on the results of the present study, we suggest that BDNF can activate BV2 microglia directly.

\section{Discussion}

BDNF was first discovered in 1982. There are two types of BDNF: pro-BDNF and mature BDNF, which act via different mechanisms in the CNS. Mature BDNF can bind to TrkB with high specificity as well as to the low-affinity receptor p75. Most biological actions of BDNF are exerted through TrkB receptors, leading to the subsequent modulation of enzymatic activity, including mitogen-activated protein kinase (MAPK), phosphatidylinositol-3 kinase (PI3K) and phospholipase $\mathrm{C}_{\gamma}\left(\mathrm{PLC}_{\gamma}\right)$.

Neuropathic pain is a debilitating chronic pain condition that results from injury to the nervous system and may be caused by cancer, diabetes mellitus, infection, autoimmune diseases, or traumatic injury [16]. BDNF is thought to promote neuronal survival and differentiation. In 1996, Lu et al. suggested that BDNF might regulate long-term potentiation (LTP) in the developing and adult hippocampus by enhancing synaptic responses to tetanic stimulation [17]. Researchers subsequently began to focus on the effect of BDNF on neuropathic pain. Substantial evidence suggests that BDNF plays a key role in synaptic transmission by potentiating the NMDA receptors present in primary afferents [18], regulating the pattern of expression and the level of activity of the transducer channels TRPA1 and TRPV1 [19], and reducing $\mathrm{Ca}^{2+}$-activated $\mathrm{K}^{+}$channel activity in the dorsal root ganglion (DRG) [20]. In our previous study, we demonstrated that BDNF could directly activate astrocytes in the spinal dorsal horn in a rat model of neuropathic pain [14].

BDNF can not only directly activate neurons and astrocytes but can also induce microglia activation. BDNF can regulate the proliferation and survival of cultured microglia [21]. Mizoguchi et al. demonstrated that BDNF could induce the sustained elevation of intracellular $\mathrm{Ca}^{2+}$ in vitro [22]. Zhou et al. performed several series of experiments to examine the effects of BDNF on microglia in an in vivo rat model and concluded that BDNF can activate spinal microglia, thereby contributing to long-term potentiation and mechanical hypersensitivity of spinal neurons [15]. Notably, in the study by Nakajima and Mizoguchi, pretreatment with BDNF significantly suppressed the release of NO from microglia activated by LPS or IFN- $\gamma[22,23]$. However, in our study, BDNF induced an increase of TNF- $\alpha$ secretion from BV2 cells. This inconsistency between our study and previous research may be the result of the method of BDNF administration. In our study, we used BDNF to stimulate microglia directly; however, in the previously discussed experiments, BDNF pretreatment was followed by LPS or IFN- $\gamma$ stimulation. We suggest there may be a mechanism of competitive inhibition underlying microglial activation. As the dramatic effects of BDNF in the central nervous system are elucidated, the sources of BDNF have received increased researcher attention. BDNF can be synthetized and secreted by neurons, astrocytes and microglia [24, 25]. Microglia-derived BDNF is a critical microglia-neuron signaling molecule that gates aberrant nociceptive processing in the spinal cord [26].

In recent years, microglia activation has begun to attract greater attention in CNS disease research. When CNS homeostasis is disrupted, microglia adopt an activated state, which stimulates proliferation, migration, and the release of proinflammatory factors. The early-stage microglial activation plays a key role in the maintenance of CNS homeostasis. 


\section{Cellular Physiology and Biochemistry}

Cell Physiol Biochem 2014;34:715-723

\begin{tabular}{l|l}
\hline DOI: $10.1159 / 000363036$ & C 2014 S. Karger AG, Basel
\end{tabular}

www.karger.com/cpb

Zhang et al.: Autocrine Activation of Microglia by BDNF

However, persistent disruption of CNS microglial homeostasis is detrimental, and further microglial activation becomes harmful. Activated microglia release abundant amounts of proinflammatory cytokines, promoting a pathological condition. However, the mechanism that triggers the transformation of beneficial activated microglia to harmful, persistently activated microglia remains unclear.

Because positive feedback often lies at the heart of pathological conditions, we performed this series of experiments to confirm that microglial-derived BDNF could further activate microglia in an autocrine manner. In 2009, studies by Trangs et al. provided a unifying mechanism for pain hypersensitivity after peripheral nerve injury through a $\mathrm{P}_{2} \mathrm{X}_{4}$ purinoceptor $\left(\mathrm{P}_{2} \mathrm{X}_{4} \mathrm{R}\right)$-evoked increase in $\mathrm{Ca}^{+}$and the subsequent activation of p38-MAPK, leading to the synthesis and exocytotic release of BDNF from microglia [12]. However, research focusing on the function of BDNF as an autocrine stimulator of microglia remains lacking. Our recent study indicates that activated microglia can produce additional BDNF, and elevated levels of BDNF will, in turn, induce additional microglial activation. This positive feedback loop results in prolonged microglial activation, which may contribute to neuropathic pain. Notably, autocrine TNF- $\alpha$ derived from microglia can also subsequently activate microglia [27]. The proinflammatory cytokine positive feedback loop may function as a trigger for the development of many pathological conditions. Future experiments will be needed to elucidate the detailed mechanism underlying the relationship between these positive feedback loops and pathological processes such as neuropathic pain. Furthermore, targeting the BDNF pathway may provide a novel therapeutic strategy for pain management.

\section{Acknowledgements}

This work was supported in part by the General Program of National Natural Science Foundation of China (81370933) and Basic Foundation of Shanghai Jiao Tong University Affiliated Sixth Hospital (1438).

\section{References}

1 Sarzi-Puttini P, Vellucci R, Zuccaro SM, Cherubino P, Labianca R, Fornasari D: The appropriate treatment of chronic pain. Clin Drug Investig 2012;32:S21-33.

$\longrightarrow 2$ Eyo UB, Dailey ME: Microglia: key elements in neural development, plasticity, and pathology. J Neuroimmune Pharmacol 2013;8:494-509.

-3 Tsuda M, Masuda T, Tozaki-Saitoh H, Inoue K: Microglial regulation of neuropathic pain. J Pharmacol Sci 2013;121:89-94.

4 Ristoiu V: Contribution of macrophages to peripheral neuropathic pain pathogenesis. Life Sci 2013;93:870881.

5 Tsuda M, Shigemoto-Mogami Y, Koizumi S, Mizokoshi A, Kohsaka S, Salter MW, Inoue K: P2X4 receptors induced in spinal microglia gate tactile allodynia after nerve injury. Nature 2003;424:778-783.

-6 Winkelstein BA, DeLeo JA: Nerve root injury severity differentially modulates spinal glial activation in a rat lumbar radiculopathy model: considerations for persistent pain. Brain Res 2002;956:294-301.

7 Kunori S, Matsumura S, Okuda-Ashitaka E, Katano T, Audoly LP, Urade Y, Ito S: A novel role of prostaglandin E2 in neuropathic pain: blockade of microglial migration in the spinal cord. Glia 2011;59:208-218.

-8 Inoue K, Koizumi S, Tsuda M, Shigemoto-Mogami Y: Signaling of ATP receptors in glia-neuron interaction and pain. Life Sci 2003;74:189-197.

9 Coull JA, Beggs S, Boudreau D, Boivin D, Tsuda M, Inoue K, Gravel C, Salter MW, De Koninck Y: BDNF from microglia causes the shift in neuronal anion gradient underlying neuropathic pain. Nature 2005;438:10171021. 


\section{Cellular Physiology and Biochemistry}

Cell Physiol Biochem 2014;34:715-723

\begin{tabular}{l|l}
\hline DOI: $10.1159 / 000363036$ & (c) 2014 S. Karger AG, Basel
\end{tabular}

Zhang et al.: Autocrine Activation of Microglia by BDNF

$\$ 10$ Huang EJ, Reichardt LF: Neurotrophins: roles in neuronal development and function. Annu Rev Neurosci 2001;24:677-736.

11 Biggs JE, Lu VB, Stebbing MJ, Balasubramanyan S, Smith PA: Is BDNF sufficient for information transfer between microglia and dorsal horn neurons during the onset of central sensitization? Mol Pain 2010;6:44.

-12 Trang T, Beggs S, Wan X, Salter MW: P2X4-receptor-mediated synthesis and release of brain-derived neurotrophic factor in microglia is dependent on calcium and p38-mitogen-activated protein kinase activation. J Neurosci 2009;29:3518-3528.

$\checkmark 13$ Bardoni R, Ghirri A, Salio C, Prandini M, Merighi A: BDNF-mediated modulation of GABA and glycine release in dorsal horn lamina II from postnatal rats. Dev Neurobiol 2007;67:960-975.

14 Zhang X, Wang J, Zhou Q, Xu Y, Pu S, Wu J, Xue Y, Tian Y, Lu J, Jiang W, Du D: Brain-derived neurotrophic factor-activated astrocytes produce mechanical allodynia in neuropathic pain. Neuroscience 2011;199:452460 .

15 Zhou LJ, Yang T, Wei X, Liu Y, Xin WJ, Chen Y, Pang RP, Zang Y, Li YY, Liu XG: Brain-derived neurotrophic factor contributes to spinal long-term potentiation and mechanical hypersensitivity by activation of spinal microglia in rat. Brain Behav Immun 2011;25:322-334.

16 Woolf CJ: Central sensitization: implications for the diagnosis and treatment of pain. Pain 2011;152:S2-15.

17 Figurov A, Pozzo-Miller LD, Olafsson P, Wang T, Lu B: Regulation of synaptic responses to high-frequency stimulation and LTP by neurotrophins in the hippocampus. Nature 1996;381:706-709.

18 Chen W, Walwyn W, Ennes HS, Kim H, McRoberts JA, Marvizon JC: BDNF released during neuropathic pain potentiates NMDA receptors in primary afferent terminals. Eur J Neurosci 2014;10.1111/ejn.12516

-19 Ciobanu C, Reid G, Babes A: Acute and chronic effects of neurotrophic factors BDNF and GDNF on responses mediated by thermo-sensitive TRP channels in cultured rat dorsal root ganglion neurons. Brain Res 2009;1284:54-67.

20 Cao XH, Chen SR, Li L, Pan HL: Nerve injury increases brain-derived neurotrophic factor levels to suppress BK channel activity in primary sensory neurons. J Neurochem 2012;121:944-953.

21 Zhang J, Geula C, Lu C, Koziel H, Hatcher LM, Roisen FJ: Neurotrophins regulate proliferation and survival of two microglial cell lines in vitro. Exp Neurol 2003;183:469-481.

22 Mizoguchi Y, Monji A, Kato T, Seki Y, Gotoh L, Horikawa H, Suzuki SO, Iwaki T, Yonaha M, Hashioka S, Kanba S: Brain-derived neurotrophic factor induces sustained elevation of intracellular Ca2+ in rodent microglia. J Immunol 2009;183:7778-7786.

23 Nakajima K, Kikuchi Y, Ikoma E, Honda S, Ishikawa M, Liu Y, Kohsaka S: Neurotrophins regulate the function of cultured microglia. Glia 1998;24:272-289.

-24 Lindholm D, Castren E, Hengerer B, Zafra F, Berninger B, Thoenen H: Differential Regulation of Nerve Growth Factor (NGF) Synthesis in Neurons and Astrocytes by Glucocorticoid Hormones. Eur J Neurosci 1992;4:404-410.

-25 Rudge JS, Alderson RF, Pasnikowski E, McClain J, Ip NY, Lindsay RM: Expression of Ciliary Neurotrophic Factor and the Neurotrophins-Nerve Growth Factor, Brain-Derived Neurotrophic Factor and Neurotrophin 3-in Cultured Rat Hippocampal Astrocytes. Eur J Neurosci 1992;4:459-471.

-26 Trang T, Beggs S, Salter MW: Brain-derived neurotrophic factor from microglia: a molecular substrate for neuropathic pain. Neuron Glia Biol 2011;7:99-108.

27 Kuno R, Wang J, Kawanokuchi J, Takeuchi H, Mizuno T, Suzumura A: Autocrine activation of microglia by tumor necrosis factor-alpha. J Neuroimmunol 2005;162:89-96. 